\title{
Proteome profile and functional classification of proteins in Arabidopsis thaliana (Landsberg erecta) mature pollen
}

\author{
Inder S. Sheoran · Kerry A. Sproule • \\ Douglas J. H. Olson · Andrew R. S. Ross • \\ Vipen K. Sawhney
}

Received: 16 May 2006/ Accepted: 15 July 2006/Published online: 11 August 2006

(C) Springer-Verlag 2006

\begin{abstract}
Proteome analysis of mature Arabidopsis thaliana (Landsberg erecta ecotype) pollen was conducted using two-dimensional gel electrophoresis and mass spectrometry. A total of 960 spots were resolved on $\mathrm{pH}$ 4-7 IPG strips and 110 distinct proteins were identified from 150 spots analyzed. The identified proteins were categorized based on their functional role in the pollen, which included proteins involved in energy regulation, defense-related mechanisms, calcium-binding and signaling, cytoskeletal formation, pollen allergens, glycine-rich proteins (GRPs), and late embryogenesis abundant (LEA) proteins. These proteins potentially play important roles in pollen function at maturity and during subsequent germination and tube growth. Some of the proteins identified were related to known pollen-specific transcripts, while some were similar to proteins found in the seed. In this study, 66 new proteins were identified which were not reported in two other recent studies on Arabidopsis pollen, 17 proteins were common in all three studies, and 35 or 26 proteins reported here had an overlap with one or the other two studies. These differences may be attributed to the methods of protein extraction,
\end{abstract}

Communicated by S. Russell

I. S. Sheoran · K. A. Sproule · V. K. Sawhney $(\bowtie)$ Department of Biology, University of Saskatchewan, 112 Science Place, Saskatoon, SK, Canada S7N 5E2 e-mail: sawhney@admin.usask.ca

D. J. H. Olson · A. R. S. Ross

National Research Council of Canada,

Plant Biotechnology Institute, 110 Gymnasium Place,

Saskatoon, SK, Canada S7N 0W9 spot selection for analysis, and the ecotype used. Together, the three studies provide a broad spectrum of the Arabidopsis pollen proteome.

Keywords Arabidopsis thaliana $\cdot$ Calcium-binding proteins $\cdot$ LEA proteins $\cdot$ Pollen $\cdot$ Proteomics

\section{Introduction}

The pollen grain plays an essential role in sexual reproduction of higher plants, with its primary function of delivering sperm cells to the female gametophyte via the formation of a pollen tube. In angiosperms, the pollen grain is a relatively simple two- or three-celled structure produced in the anther of a stamen. The development of pollen is highly controlled and follows a precise pattern of sporogenesis and gametogenesis, with some differences between dicots and monocots at the time of meiosis of pollen mother cells (Bedinger 1992; Palmer et al. 1992).

Genetic control of anther and pollen development has been investigated in a number of species, including Arabidopsis (Mascarenhas 1990). A large number of genes controlling stamen and pollen development have been identified using a variety of approaches, including mutant isolation, mRNA expression, and in situ hybridization (Sanders et al. 1999; McCormick 2004; Scott et al. 2004). Both nuclear and cytoplasmic (mostly mitochondrial) genes regulate pollen development (Hanson and Betolila 2004), and approximately 20,000 genes are expressed during this process of which about $10 \%$ are specifically expressed in the pollen (Hamilton and Mascarenhas 1997; Honys and Twell 2003). Detailed transcriptomic analyses of Arabidopsis pollen 
have revealed that a majority of genes expressed in the pollen have an overlap with sporophytic tissues, but several genes are either enriched or specifically expressed in the pollen (Becker et al. 2003; Honys and Twell 2003; Pina et al. 2005). As well, the most prevalent pollen-expressed genes are involved in signaling, vesicle transport, and the cytoskeleton.

We extend these studies to analyze the Arabidopsis pollen proteome and to classify proteins based on their functional roles. An understanding of the pollen proteome would provide insight into the relationship between the transcriptome in mature pollen and certain specialized proteins that are readily available for pollen germination and tube growth. In addition, since the pollen grain, like the seed, is a dispersal agent of higher plants that is capable of becoming highly desiccated and dormant, there are possible similarities between the pollen (Holmes-Davis et al. 2005; Noir et al. 2005; Dai et al. 2006) and seed (Gallardo et al. 2001; Sheoran et al. 2005; Vensel et al. 2005) that are reflected in the proteome.

Two articles have recently appeared on the Arabidopsis pollen proteome in which different methods of protein extraction were applied to the Columbia ecotype (Holmes-Davis et al. 2005; Noir et al. 2005). Although some of the identified proteins are common between these studies, many proteins are different in at least two of the three studies. Taken together, the results of these three independent investigations provide a firm basis of technological and biological sampling and provide a broad analysis of the Arabidopsis pollen proteome. This study also reports on a number of proteins that were not reported earlier in Arabidopsis pollen.

\section{Materials and methods}

Plant material and growth conditions

Seeds of the wild type Arabidopsis thaliana ecotype Landsberg erecta were originally provided by Dr. B. Mulligan of the University, Nottingham, UK (Fei et al. 2004). Seeds were placed in $15 \mathrm{~cm}$ plastic pots containing Tera-lite Redi-earth mix and were stratified by exposing the pots to $15^{\circ} \mathrm{C}$ in the dark for 3 days. Plants were subsequently grown at $22 / 20^{\circ} \mathrm{C}$ (day/night) and $16 / 8 \mathrm{~h} \mathrm{light/dark}$ in a growth chamber. Light was provided by fluorescent tubes (Osram Sylvania Versailes, KY, USA) at $120-150 \mu \mathrm{M} \mathrm{m}^{-2} \mathrm{~s}^{-1}$.

Pollen collection

Flowers at anthesis were picked daily, and placed in open micro-centrifuge tubes overnight at $4^{\circ} \mathrm{C}$ to pro- mote anther dehiscence. Flowers were then rinsed at least four times with acetone in the tubes to obtain a pollen suspension, which was transferred to fresh tubes using a pipette. The pollen suspension was centrifuged, the supernatant removed, and the pellet transferred onto a glass slide. Pollen samples were checked under a dissecting microscope, and pooled following the removal of any debris. The purity of isolated pollen was determined by light microscopy, and the pollen was stored at $-80^{\circ} \mathrm{C}$ until further use. Two separate batches of clean and pooled samples were used for protein extraction. Pollen viability of each of the two batches of freshly colleted pollen was tested by in vitro pollen germination test (Shivanna and Sawhney 1995) and it ranged from 70 to $75 \%$.

\section{Protein extraction}

The pollen (approximately $20 \mathrm{mg}$ ) was ground with a glass rod in a micro-centrifuge tube with cold $10 \%$ TCA and $1 \%$ DTT in acetone and kept at $-20^{\circ} \mathrm{C}$ for at least $2 \mathrm{~h}$. The samples were centrifuged at $25,000 \mathrm{~g}$ for $20 \mathrm{~min}$ at $4^{\circ} \mathrm{C}$, and the resulting pellet was washed by suspending in acetone containing 1\% DTT, incubated at $-20^{\circ} \mathrm{C}$ for $2 \mathrm{~h}$, and centrifuged. The pellet was suspended again in acetone, sonicated $(3 \times 15 \mathrm{~s})$, and centrifuged at $25,000 \mathrm{~g}$. The pellet was vacuum dried and total soluble proteins were extracted by dissolving in iso-electric focusing (IEF) compatible buffer comprising $8 \mathrm{M}$ urea, $20 \mathrm{mM}$ DTT, $4 \%$ CHAPS, and $2 \%$ ampholyte (pH 3-10). The solution was vortexed extensively for $1 \mathrm{~h}$ at room temperature, centrifuged at $20^{\circ} \mathrm{C}$ for $20 \mathrm{~min}$ at $25,000 \mathrm{~g}$, and the supernatant was collected. The resulting pellet was resolubilized and vortexed for $1 \mathrm{~h}$, centrifuged at $25,000 \mathrm{~g}$, and the supernatant combined with that collected earlier. The resulting protein samples were centrifuged again for $20 \mathrm{~min}$ at $25,000 \mathrm{~g}$. Total soluble protein in the supernatant was estimated with Bio-Rad protein assay (Bio-Rad, Hercules, CA, USA) and used immediately for further analysis or stored at $-80^{\circ} \mathrm{C}$ for later use. The protein content in the samples ranged between 95 and $120 \mu \mathrm{g} / \mathrm{mg}$ fresh wt.

Two-dimensional gel electrophoresis

Two-dimensional electrophoresis (2-DE) was conducted according to Sheoran et al. (2005). IEF was performed using the Multiphor II horizontal electrophoresis system and $13 \mathrm{~cm}$ Immobiline Dry Strips of 4-7 or 3-10 linear $\mathrm{pH}$ gradient (Amersham Pharmacia Biotech, Uppsala, Sweden). The strips were rehydrated overnight in a solution containing $8 \mathrm{M}$ urea, 
2\% CHAPS, $20 \mathrm{mM}$ DTT, $0.002 \%$ bromophenol blue, $2 \%$ IPG buffer ( $\mathrm{pH} 3-10)$, and $600 \mu \mathrm{g}$ of the protein sample. IEF was carried out by applying a voltage of $250 \mathrm{~V}$ for $1 \mathrm{~h}$, increasing to $3,500 \mathrm{~V}$ over $2 \mathrm{~h}$, and holding at $3,500 \mathrm{~V}$ until total of $75 \mathrm{kVh}$ was obtained.

Following IEF, the strips were equilibrated for $15 \mathrm{~min}$ in an equilibration buffer containing $0.05 \mathrm{M}$ Tris- $\mathrm{HCl}(\mathrm{pH} 8.8), 6 \mathrm{M}$ urea, $30 \%(\mathrm{v} / \mathrm{v})$ glycerol, $2 \%$ $(\mathrm{w} / \mathrm{v})$ SDS, and $0.125 \%(\mathrm{w} / \mathrm{v})$ DTT, followed by another $15 \mathrm{~min}$ equilibration in the same buffer containing $125 \mathrm{mM}$ iodoacetamide without DTT. The equilibrated strips were applied to vertical SDS-polyacrylamide gels (12.5\% resolving 5\% stacking) and sealed with $0.5 \%$ agarose in SDS buffer containing bromophenol blue. Electrophoresis was performed for $30 \mathrm{~min}$ at $25 \mathrm{~mA}$, and then for $3 \mathrm{~h}$ at $40 \mathrm{~mA}$ in SDS electrophoresis buffer containing $25 \mathrm{mM}$ Tris base, $192 \mathrm{mM}$ glycine, and $0.1 \%$ SDS, $\mathrm{pH} 8.3$.

\section{Gel staining}

Gels were fixed in $50 \%$ ethanol with $10 \%$ orthophosphoric acid over-night, washed with water $(3 \times 20 \mathrm{~min})$, and stained in a Colloidal Coomassie Blue G-250 (CCB) solution $(0.12 \%$ CB, $10 \%$ ammonium sulfate, $10 \%$ orthophosphoric acid, $20 \%$ methanol; Candiano et al. 2004) for 2 days. After washing with water, gels were scanned, annotated, and analyzed for spot number and spot volume using Phoretix 2D Image analysis software (UBI, Canada). Three replicate gels were run for each of two different pollen samples, and protein spots observed consistently in replicate gels were selected for further analysis.

\section{Mass spectrometry}

Of the 960 spots observed consistently in stained gels, 150 spots were selected across the gel representing different molecular weights and PIs for identification by mass spectrometry. These spots were excised using a Protean 2-D spot cutter (Bio-Rad), and placed in a 96well microtitre plate (Sigma, Milwaukee, WI, USA). Excised proteins were automatically de-stained, dehydrated, reduced with DTT, alkylated with iodoacetamide, and digested with trypsin using a MassPREP protein digest station according to the recommended procedures (Micromass, Manchester, UK). Mass spectra of the resulting tryptic digests were acquired by matrix-assisted laser desorption/ionization-time of flight mass spectrometry (MALDI-TOF MS) on a Voyager-DE STR (Applied Biosystems, Framingham, MA, USA) as previously described (Sheoran et al. 2005). Five micro liter of each digest were dried to approximately $1 \mu \mathrm{l}$ on a MALDI target plate. One micro liter of $\alpha$-cyano- 4 hydroxy-cinnamic acid matrix solution $(5 \mathrm{mg} / \mathrm{ml}$ in $0.1 \% \mathrm{TFA} / 75 \%$ acetonitrile) was then added to each sample, and the mixture allowed to air dry. The instrument was calibrated using trypsin autolysis products $(\mathrm{m} / \mathrm{z}, 842.51$ and $2,211.10)$ as internal standards, or a mixture of des-Arg bradykinin $(\mathrm{m} / \mathrm{z}$ 904.4681) and ACTH clip 18-39 ( $\mathrm{m} / z, 2,465.1989)$ for close external calibration. Peak lists generated from the tryptic digest spectra were submitted for peptide mass fingerprinting (PMF) using MASCOT (http:// www.matrixscience.com/) search engine and NCBI non-redundant protein database (November, 2005). The following parameters were used for database searching: carbamidomethylation of cysteine (fixed modification); oxidation of methionine (variable modification); one missed cleavage (trypsin); and a mass deviation of less than $50 \mathrm{ppm}$. For proteins that could not be identified by PMF, the remainder of the digest was analyzed by liquid chromatography-tandem mass spectrometry (LC-MS/MS) using a capLC system and Q-Tof Ultima Global mass spectrometer (Waters-Micromass, Milford, MA, USA) as previously described (Sheoran et al. 2005). LC-MS/MS data were processed using ProteinLynx v2.15 software (Micromass) and searched against NCBI non-redundant protein database using the MASCOT search engine. Proteins identified with a MOWSE score greater than 70 (95\% confidence interval) are reported. Functional categorization and sub-cellular localization of identified proteins was performed using The Arabidopsis Information Resource (TAIR) database (http://www.arabidopsis.org).

\section{Results and discussion}

On average, 960 spots were observed on a 2-D gel stained with CCB using $\mathrm{pH} 4-7$ IPG strips (Fig. 1). Of the 150 spots analyzed using both MALDI-TOF MS and LC-MS/MS, 132 spots representing 110 distinct proteins were identified by searching various publicly available protein databases. Table 1 lists each identified protein by its gene index (gi) number and the corresponding AGI gene locus, as obtained from TAIR. In most cases, the calculated molecular mass and $\mathrm{pI}$ of identified proteins were close to the theoretical values (Table 1). Multiple spots corresponding to the same protein were also identified, as has been reported in other proteomic studies. Deviations in molecular mass and pI, and the presence of multiple spots corresponding to the same protein, could be due to a number of factors, including post-translational 
Fig. 1 Colloidal Coomassie stained 2-DE gel ( $\mathrm{pH} \mathrm{4-7)}$ of mature Arabidopsis pollen. Spot numbers indicated on the gel were subjected to MALDI and/or LC- MS/MS analysis. Standard molecular weight markers are shown on the right

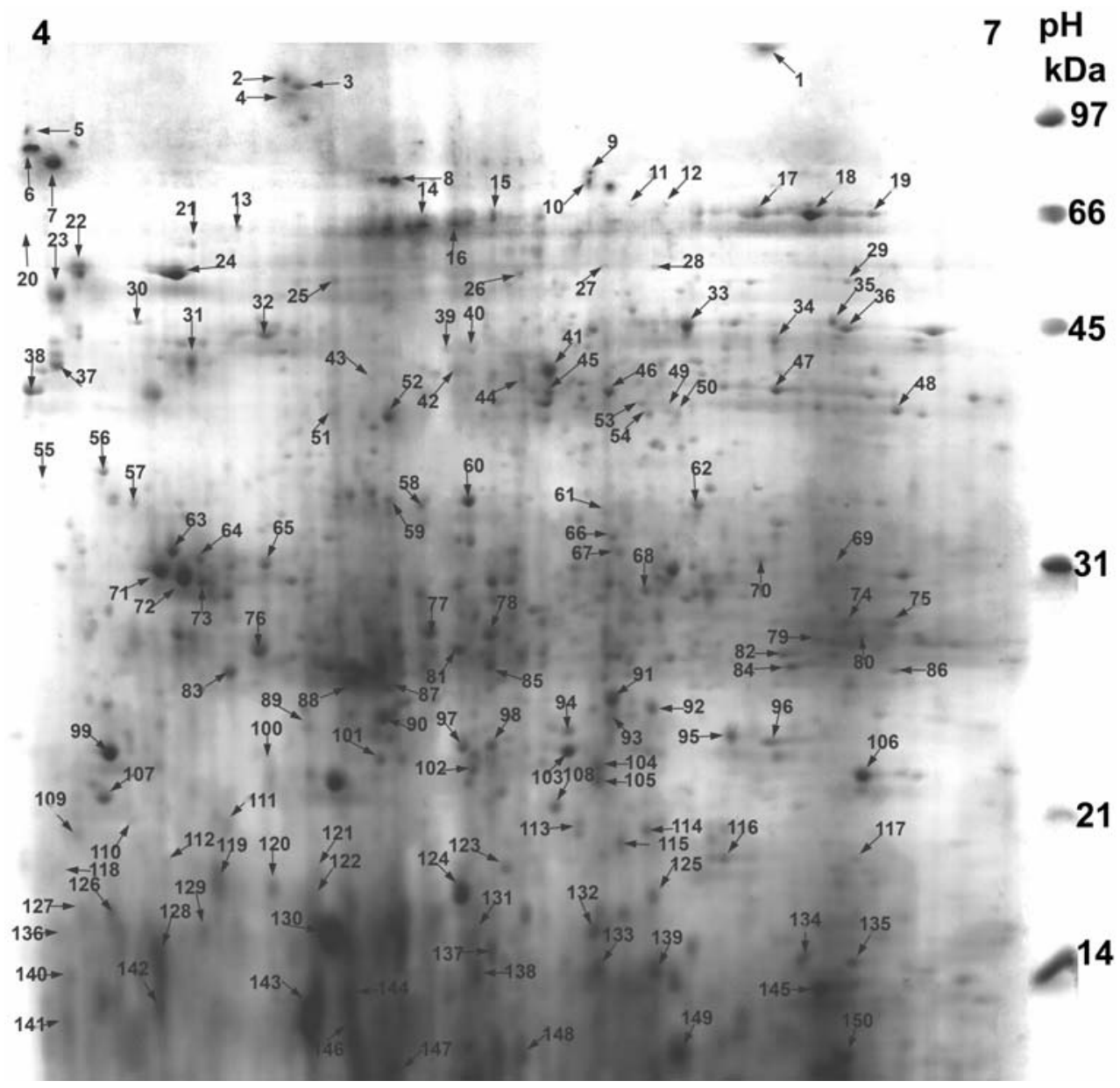

modifications (e.g., subsequent addition of phosphate, methyl, or other groups), protein degradation, partial synthesis of proteins during pollen maturation, or protein translation from alternatively spliced mRNAs.

Pollen is an abundant source of protein, with protein content ranging from 2.5 to $61 \%$ (Roulston et al. 2000). Pollen proteins identified in this study are likely to be predominent in the vegetative cell, since sperm cells contain few organelles and a small cellular volume, e.g., less than $0.05 \%$ of the pollen volume in Plumbago (Russell and Strout 2005), and proportionately fewer transcript copies (Xu et al. 1999; Engel et al. 2003). Using the Gene Ontology annotation for Arabidopsis (Berardini et al. 2004), the relative distribution of identified proteins (Table 1) associated with various cell components is presented in Fig. 2. Mitochondrial proteins accounted for the largest single group (approximately 29\%), followed by proteins in the cytosol (10\%). Other cellular sources include ER (7\%), nucleus $(6 \%)$, and cell wall $(5 \%)$. About $19 \%$ of protein products could not be assigned to a specific cellular component. Noir et al. (2005) reported that the largest protein fraction in the pollen of Arabidopsis
Columbia ecotype to be cytoplasmic (41\%), with $40 \%$ targeted to the endomembrane system. We observed more pollen proteins associated with the cell wall than did Noir et al. (2005), which may, in part, be due to different methods of protein extraction.

Functional classification of identified proteins

The identified proteins (Table 1) were classified into functional groups (Fig. 3) based on putative protein functions (Berardini et al. 2004). The two major groups of identified proteins in Arabidopsis pollen were those involved in energy regulation $(18 \%)$ and those involved in defense- and stress- response $(20 \%)$. Other well-represented functional categories were protein and other metabolism (9\% each), cytoskeleton $(8 \%)$, transport $(8 \%)$, and transcriptional regulation (4\%). We separated $\mathrm{Ca}^{2+}$-binding and signaling $(5 \%)$, pollen allergen $(6 \%)$, and LEA proteins $(5 \%)$ into separate groups because of their physiological significance in the pollen. The other two recent studies (Holmes-Davis et al. 2005; Noir et al. 2005) also suggest that the majority of proteins 
Table 1 Arabidopsis (Landsberg erecta) mature pollen proteins identified by MALDI-TOF MS or LC-MS/MS

\begin{tabular}{|c|c|c|c|c|c|c|}
\hline $\begin{array}{l}\text { Spot } \\
\text { number }^{\mathrm{a}}\end{array}$ & $\begin{array}{l}\text { Gene index } \\
\text { number }\end{array}$ & $\begin{array}{l}\text { AGI gene } \\
\text { locus }\end{array}$ & Protein identity & $\mathrm{kDa} / \mathrm{pI}^{\mathrm{b}}$ & $\begin{array}{l}\text { Sequence } \\
\text { coverage }(\%)\end{array}$ & $\begin{array}{l}\text { Mascot } \\
\text { score }\end{array}$ \\
\hline 1 & 7268300 & At4g15530 & Pyruvate phosphate dikinase family protein ${ }^{\mathrm{d}}$ & $104.9 / 5.8$ & 23 & 108 \\
\hline 2 & 15222149 & At1g49490 & Structural constituent of cell wall & $91.2 / 4.9$ & 28 & 147 \\
\hline 3 & 15222149 & At1g49490 & Structural constituent of cell wall & $91.2 / 4.9$ & 23 & 69 \\
\hline 4 & 15222149 & At1g49490 & Structural constituent of cell wall & $91.2 / 4.9$ & 15 & 89 \\
\hline 5 & 30681829 & At2g22795 & Unknown protein & $82.0 / 4.3$ & 45 & 354 \\
\hline 6 & 30681829 & At2g22795 & Unknown protein & $82.0 / 4.3$ & 30 & 210 \\
\hline 7 & 6681343 & At3g09840 & Cell division cycle protein $48^{\mathrm{c}, \mathrm{d}}$ & $89.3 / 5.0$ & 65 & 413 \\
\hline 8 & 1303695 & At5g28540 & Luminal binding protein $(\mathrm{BiP})^{\mathrm{c}}$ & $73.7 / 5.1$ & 40 & 1,029 \\
\hline 9 & 18414718 & At4g16660 & ATP binding & $96.7 / 5.9$ & 19 & 295 \\
\hline 10 & 15293149 & At1g11660 & HSP protein & $96.9 / 5.8$ & 15 & 250 \\
\hline 11 & 30697452 & At5g61030 & RNA binding/protein transporter & $49.4 / 5.7$ & 33 & 360 \\
\hline 12 & 21553535 & At5g60980 & Ras-GTPase-activating protein (NTF2) & $49.4 / 5.7$ & 41 & 405 \\
\hline 13 & 15223975 & At1g77510 & ATPDIL1-2/disulfide isomerase & $56.6 / 4.9$ & 22 & 196 \\
\hline 14 & 11994364 & At3g13930 & Dihydrolipoamide acetyltransferase & $60.1 / 7.5$ & 13 & 76 \\
\hline 16 & 30725440 & At1g78900 & Vacuolar ATP synthase subunit $\mathrm{A}^{\mathrm{c}, \mathrm{d}}$ & $68.8 / 5.0$ & 66 & 291 \\
\hline 17 & 10177326 & At5g65690 & Phosphoenol- pyruvate carboxykinase ${ }^{\mathrm{d}}$ & $68.7 / 6.0$ & 44 & 186 \\
\hline 19 & 5107826 & At5g 25880 & Putative malate oxidoreductase $\mathrm{e}^{\mathrm{c}, \mathrm{d}}$ & $64.6 / 6.6$ & 26 & 164 \\
\hline 20 & 30696733 & At5g56360 & Calmodulin binding & $74.1 / 4.7$ & 10 & 98 \\
\hline 21 & 15219086 & At1g21750 & ATPDIL1-1/disulfide isomerase ${ }^{\mathrm{c}, \mathrm{d}}$ & $55.8 / 4.8$ & 50 & 965 \\
\hline 22 & 30725696 & At1g56340 & Calreticulin ${ }^{\mathrm{d}}$ & $48.7 / 4.5$ & 47 & 642 \\
\hline 23 & 21555174 & At1g09210 & Calreticulin $^{\mathrm{d}}$ & $48.4 / 5.9$ & 54 & 611 \\
\hline 24 & 23308191 & At5g39570 & Expressed protein (glycine rich protein) & $43.6 / 4.7$ & 42 & 178 \\
\hline $25^{*}$ & 52354239 & At1g79120 & Hypothetical protein (DUF 860 domain) & $47.5 / 6.0$ & 27 & 67 \\
\hline 26 & 13548325 & At5g08670 & ATP synthase beta-chain ${ }^{c}$ & $59.8 / 6.2$ & 48 & 376 \\
\hline 27 & 20148521 & At1g23190 & Phosphoglucomutase $\mathrm{d}^{\mathrm{d}}$ & $63.1 / 5.9$ & 67 & 261 \\
\hline 28 & 20148521 & At1g23190 & Phosphoglucomutase $^{\mathrm{d}}$ & $63.1 / 5.9$ & 49 & 197 \\
\hline $29 *$ & 15221044 & At1g48030 & Dihydrolipoyl dehydrogenase (F21D18028) & $54.0 / 7.0$ & 37 & 80 \\
\hline 30 & 23308191 & At5g39570 & Unknown & $43.6 / 4.7$ & 34 & 273 \\
\hline 31 & 1303695 & At5g42020 & Luminal binding protein $(\mathrm{HSC}-70)^{\mathrm{c}}$ & $73.7 / 5.1$ & 24 & 593 \\
\hline 32 & 16323374 & At5g 44340 & Tubulin beta-4 chain ch, $^{\mathrm{c}}$ & $49.8 / 4.6$ & 39 & 106 \\
\hline 33 & 20453235 & At1g65930 & Isocitrate dehydrogenase $\mathrm{e}^{\mathrm{c}, \mathrm{d}}$ & $45.7 / 6.1$ & 44 & 179 \\
\hline $35^{*}$ & 9294498 & At3g17940 & Aldolase 1-epimerase-like protein ${ }^{\mathrm{d}}$ & $37.3 / 5.9$ & 29 & 79 \\
\hline 36 & 7529717 & At3g52930 & Fructose bisphosphate aldolase ${ }^{\mathrm{c}, \mathrm{d}}$ & $38.9 / 6.1$ & 27 & 214 \\
\hline 37 & 21555174 & At1g09210 & Calreticulin ${ }^{\mathrm{d}}$ & $48.4 / 4.4$ & 22 & 143 \\
\hline 38 & 21555174 & At1g09210 & Calreticulin $^{\mathrm{d}}$ & $48.4 / 4.4$ & 35 & 261 \\
\hline 39 & 4309733 & At2g18340 & Similar to LEA protein & $49.8 / 5.5$ & 29 & 176 \\
\hline 41 & 16226235 & At2g36530 & F1011.16/enolase $\mathrm{e}^{\mathrm{c}, \mathrm{d}}$ & $35.0 / 5.2$ & 10 & 115 \\
\hline $42 \mathrm{a}$ & 17939849 & At5g08670 & Mitochondrial ATP synthase beta subunit ${ }^{\mathrm{c}}$ & $63.6 / 6.5$ & 20 & 355 \\
\hline $42 b$ & 28393806 & At5g59370 & Actin $4^{\mathrm{d}}$ & $41.9 / 5.3$ & 16 & 188 \\
\hline 44 & 4586118 & At4g12130 & $\begin{array}{l}\text { Putative protein (glycine cleavage } \mathrm{T} \text { family/ } \\
\text { aminomethyl transferase family protein) }\end{array}$ & $40.5 / 8.7$ & 32 & 176 \\
\hline 45 & 877848 & At1g55700 & F20N2.12 (DC-domain containing protein) & $77.8 / 6.5$ & 13 & 177 \\
\hline 46 & 24417274 & At2g 47470 & Unknown protein (disulfide isomerase) $)^{\mathrm{c}, \mathrm{d}}$ & $39.8 / 5.8$ & 34 & 320 \\
\hline 47 & 4454472 & At2g20760 & Expressed protein (unknown protein) & $37.2 / 5.9$ & 25 & 102 \\
\hline 48 & 12642848 & At1g53240 & Mitochondrial NAD-dependent MDH & $36.0 / 8.5$ & 31 & 111 \\
\hline 49 & 8778388 & At1g13890 & F16A14.10 (Synaptosomal-associated protein) & $29.1 / 5.8$ & 47 & 392 \\
\hline 50 & 8778388 & At5g61210 & F16A14.10 (Synaptosomal-associated protein) & $29.1 / 5.8$ & 47 & 442 \\
\hline 51 & 21554576 & At3g53750 & Actin $3^{\mathrm{d}}$ & $41.7 / 5.2$ & 34 & 98 \\
\hline 52 & 46931236 & At4g36600 & Similar to LEA protein & $37.6 / 5.3$ & 29 & 652 \\
\hline $53 *$ & 42571269 & At2g 47470 & Thioredoxin family protein ${ }^{\mathrm{c}, \mathrm{d}}$ & $29.5 / 5.5$ & 25 & 88 \\
\hline $55^{*}$ & 20465723 & At1g22450 & Putative cytochrome-C oxidase subunit & $21.4 / 4.3$ & 23 & 76 \\
\hline 56 & 1695717 & At1g09080 & Luminal binding protein & $73.0 / 5.0$ & 24 & 268 \\
\hline 57 & 24496493 & At5g02490 & BiP chaperone BIP-L & $75.3 / 4.9$ & 12 & 362 \\
\hline 58 & 24417274 & At2g 47470 & Unknown protein (disulfide isomerase) ${ }^{\mathrm{c}, \mathrm{d}}$ & $39.9 / 5.8$ & 39 & 475 \\
\hline 59 & 24417274 & At2g 47470 & Unknown protein (disulfide isomerase) ${ }^{\mathrm{c}, \mathrm{d}}$ & $39.9 / 5.8$ & 18 & 122 \\
\hline $60 *$ & 15226610 & At2g 47470 & ATPDIL2-1 electron transporter ${ }^{\mathrm{c}, \mathrm{d}}$ & $40.0 / 5.8$ & 35 & 144 \\
\hline 61 & 8777485 & At3g15020 & NAD-dependent $\mathrm{MDH}$ & $36.0 / 8.3$ & 42 & 782 \\
\hline 62 & 7769871 & At1g53240 & F12M16.14 (NAD-dependent MDH) & $37.2 / 8.5$ & 34 & 502 \\
\hline $63^{*}$ & 2083278 & At1g35720 & Annexin $1^{\mathrm{d}}$ & $37.8 / 5.2$ & 66 & 125 \\
\hline $64 *$ & 21280929 & At5g16510 & Reversiably glycolated polypeptide ${ }^{\mathrm{d}}$ & $38.6 / 4.9$ & 39 & 75 \\
\hline
\end{tabular}


Table 1 continued

\begin{tabular}{|c|c|c|c|c|c|c|}
\hline $\begin{array}{l}\text { Spot } \\
\text { number }^{\mathrm{a}}\end{array}$ & $\begin{array}{l}\text { Gene index } \\
\text { number }\end{array}$ & $\begin{array}{l}\text { AGI gene } \\
\text { locus }\end{array}$ & Protein identity & $\mathrm{kDa} / \mathrm{pI}^{\mathrm{b}}$ & $\begin{array}{l}\text { Sequence } \\
\text { coverage }(\%)\end{array}$ & $\begin{array}{l}\text { Mascot } \\
\text { score }\end{array}$ \\
\hline 65 & 7671424 & At5g09650 & Inorganic pyrophosphatase & $33.6 / 5.7$ & 10 & 98 \\
\hline 66 & 8777485 & At3g15020 & NAD-dependent MDH & $36.0 / 8.3$ & 63 & 692 \\
\hline 68 & 16173 & At1g07890 & L-ascorbate peroxidase $(\mathrm{APX} 1)^{\mathrm{c}, \mathrm{d}}$ & $27.8 / 5.7$ & 13 & 70 \\
\hline 69 & 15225402 & At2g45080 & T14P1.11 (cyclin protein) & $26.1 / 4.4$ & 19 & 67 \\
\hline 70 & 21554322 & At1g07890 & L-ascorbate peroxidase ${ }^{c, d}$ & $27.8 / 5.7$ & 50 & 500 \\
\hline $71 *$ & 62320230 & At5g28540 & Luminal binding protein & $15.0 / 4.4$ & 39 & 75 \\
\hline $72 *$ & 62320230 & At5g28540 & Luminal binding protein & $15.0 / 4.4$ & 43 & 115 \\
\hline $73 *$ & 22006985 & At3g28340 & Glycosyl transferase & $41.0 / 4.4$ & 30 & 115 \\
\hline $74 *$ & 30690772 & At2g47730 & Glutathione S-transferase $6(\mathrm{GST} 6)^{\mathrm{c}, \mathrm{d}}$ & $29.3 / 8.5$ & 38 & 80 \\
\hline $75 \mathrm{a}$ & 2651722 & At4g13560 & Unknown protein $(\text { similar to LEA } 3)^{\mathrm{d}}$ & $11.6 / 6.7$ & 45 & 295 \\
\hline $75 b$ & 25090885 & At3g06050 & Thioredoxin reductase $e^{\mathrm{c}, \mathrm{d}}$ & $21.5 / 8.9$ & 40 & 274 \\
\hline 76 & 11270444 & At3g55440 & Triosephosphate isomerase ${ }^{\mathrm{d}}$ & $27.1 / 5.1$ & 72 & 217 \\
\hline 77 & 21554322 & At1g07890 & L-ascorbate peroxidase $\mathrm{c}^{\mathrm{c}, \mathrm{d}}$ & $27.8 / 5.7$ & 41 & 348 \\
\hline 78 & 19310625 & At2g21870 & Putative ATP synthase ${ }^{\mathrm{c}, \mathrm{d}}$ & $27.6 / 6.3$ & 30 & 475 \\
\hline $80 *$ & 21553457 & At3g10920 & Putative $(\mathrm{Mn})$ superoxide dismutase ${ }^{\mathrm{c}}$ & $25.0 / 8.5$ & 32 & 67 \\
\hline $81 *$ & 19310625 & At2g21870 & Expressed protein (ATP synthase) $)^{\mathrm{c}, \mathrm{d}}$ & $27.6 / 6.3$ & 35 & 336 \\
\hline 82 & 21593056 & At1g75270 & Dehydroascorbate reductase & $23.5 / 6.0$ & 41 & 354 \\
\hline 83 & 26452310 & At5g27980 & Embryonic abundant protein (LEA protein) & $19.6 / 4.9$ & 40 & 236 \\
\hline 84 & 18403457 & At3g22600 & Lipid binding protein & 21.1/6.1 & 12 & 88 \\
\hline 86 & 60543359 & At2g21060 & Glycine-rich protein 2 (GRP-2) & $19.4 / 6.3$ & 44 & 179 \\
\hline 87 & 21554322 & At1g07890 & L-Ascorbate peroxidase $1^{\mathrm{c}, \mathrm{d}}$ & $27.5 / 5.7$ & 60 & 284 \\
\hline 91 & 21593056 & At1g75270 & Dehydroascorbate reductase & $23.4 / 6.0$ & 23 & 770 \\
\hline 92 & 196965 & At2g47650 & dTDP-glucose 4,6-dehydratase ${ }^{c}$ & $50.0 / 8.9$ & 9 & 97 \\
\hline 95 & 18387457 & At3g06050 & Antioxidant/peroxidase $\mathrm{e}^{\mathrm{c}, \mathrm{d}}$ & $21.5 / 5.9$ & 23 & 271 \\
\hline $96 a$ & 20466103 & At3g06050 & Unknown (Alkyl hydroperoxide reductase) ${ }^{\mathrm{c}, \mathrm{d}}$ & $21.3 / 9.0$ & 34 & 147 \\
\hline $96 b$ & 6714406 & At3g05930 & Germin-like protein ${ }^{\mathrm{c}, \mathrm{d}}$ & $23.1 / 8.8$ & 15 & 135 \\
\hline 97 & 20197320 & At2g46860 & Inorganic pyrophasphatase $^{\mathrm{d}}$ & $24.9 / 5.5$ & 31 & 185 \\
\hline 99 & 26450755 & At4g20780 & Calcium-binding protein & $21.2 / 4.6$ & 38 & 301 \\
\hline 101 & 16173 & At1g07890 & L-ascrobate peroxidase $(\mathrm{APX} 1)^{\mathrm{c}, \mathrm{d}}$ & $27.8 / 5.7$ & 24 & 170 \\
\hline 102 & 30690243 & At5g26667 & Uridylate kinase $^{c}$ & $23.3 / 6.4$ & 28 & 178 \\
\hline $103 a$ & 19310625 & At2g21870 & Putative ATP synthase ${ }^{\mathrm{c}, \mathrm{d}}$ & $27.6 / 6.3$ & 30 & 457 \\
\hline $103 b$ & 12083342 & At1g16470 & Multicatalytic endopeptidase & $25.7 / 5.5$ & 48 & 275 \\
\hline 104 & 30690246 & At5g26667 & Uridylate kinase $^{c}$ & $22.6 / 5.8$ & 57 & 370 \\
\hline $105 \mathrm{a}$ & 17104709 & At1g73230 & RNA polymerase B transcription factor 3 & $18.0 / 5.9$ & 58 & 262 \\
\hline $105 b$ & 2497486 & At5g26667 & Uridylate kinase $^{c}$ & $22.6 / 5.8$ & 41 & 247 \\
\hline 106 & 7267860 & At4g11600 & Phospholipid hydroperoxide glutathione peroxidase ${ }^{\mathrm{c}, \mathrm{d}}$ & $18.7 / 6.6$ & 27 & 120 \\
\hline 107 & 1303695 & At5g42020 & Luminal binding protein $(\mathrm{HSC}-70)^{\mathrm{c}}$ & $73.7 / 5.1$ & 10 & 151 \\
\hline 108 & 14532850 & At4g38680 & Glycine-rich protein 2 (GRP-2) & $19.5 / 5.6$ & 10 & 82 \\
\hline $109^{*}$ & 42570833 & At2g20630 & Protein phosphatase $2 \mathrm{C}$ putative & $32.1 / 5.9$ & 27 & 71 \\
\hline 110 & 25403253 & At1g24620 & F21J9.28 (similar to polcalcin) & $20.4 / 4.6$ & 71 & 398 \\
\hline 111 & 48310598 & At4g02550 & Pectin methylesterase inhibitor (PMEI) & $20.0 / 4.9$ & 21 & 76 \\
\hline 112 & 25403253 & At1g24620 & F21J9.28 (similar to polcalcin) & $20.4 / 4.6$ & 27 & 110 \\
\hline 113 & 20197312 & At2g47730 & Glutathione S-transferase ${ }^{\mathrm{c}, \mathrm{d}}$ & $19.8 / 5.3$ & 28 & 195 \\
\hline 114 & 11762128 & At4g17530 & Ras-related GTP-binding protein $(\mathrm{RAB} 1 \mathrm{C})^{\mathrm{c}}$ & $24.8 / 5.8$ & 23 & 72 \\
\hline 115 & 26452507 & At5g16450 & $\begin{array}{l}\text { S-adenosylmethionine 2-dimethylmenaquinone } \\
\text { methyltransferase }\end{array}$ & $18.1 / 5.4$ & 31 & 129 \\
\hline 116 & 21593482 & At4g24640 & Pectin methylesterase inhibitor $(\text { Bnm1like protein })^{\mathrm{c}, \mathrm{d}}$ & $20.1 / 5.5$ & 66 & 351 \\
\hline 117 & 7267860 & At4g11600 & Phospholipid hydroperoxide glutathione peroxidase $e^{c, d}$ & $18.7 / 6.6$ & 27 & 143 \\
\hline 118 & 15221781 & At1g24620 & Calcium-binding pollen allergen (polcalcin) & $20.4 / 4.6$ & 27 & 110 \\
\hline 119 & 16203 & At5g20230 & Blue-copper binding protein ${ }^{\mathrm{c}}$ & $20.2 / 4.7$ & 9 & 89 \\
\hline 120 & 17939851 & At3g52300 & Putative protein (ATP synthase D chain $)^{\mathrm{d}}$ & $19.6 / 5.1$ & 11 & 83 \\
\hline 121 & 21555349 & At3g52300 & F0-ATP Synthase D chain ${ }^{\mathrm{d}}$ & $19.6 / 5.1$ & 62 & 515 \\
\hline $122 \mathrm{a}$ & 21553949 & At2g30410 & Tubulin folding cofactor $\mathrm{A}$ & $12.9 / 5.0$ & 42 & 310 \\
\hline $122 b$ & 21555349 & At3g52300 & F0-ATP Synthase D chain ${ }^{\mathrm{d}}$ & $19.6 / 5.1$ & 59 & 296 \\
\hline 123 & 4914438 & At2g21690 & Glycine-rich RNA binding protein 8 & $16.5 / 5.6$ & 39 & 270 \\
\hline $124 *$ & 4914438 & At2g21690 & Glycine-rich RNA binding protein 8 & $16.5 / 5.6$ & 51 & 72 \\
\hline $125^{*}$ & 21537051 & At1g26630 & Initiation factor $5 \mathrm{~A}-2$ & $17.4 / 5.6$ & 33 & 76 \\
\hline 126 & 21553555 & At2g22170 & Lipoxygenase (dehydration stress-induced) & $20.3 / 5.1$ & 9 & 77 \\
\hline 127 & 228408 & At1g66400 & Calmodulin 1 & $15.5 / 4.2$ & 65 & 262 \\
\hline 129 & 2021593095 & At2g27710 & $60 S$ ribosomal protein $\mathrm{P} 2$ & $11.4 / 4.8$ & 23 & 198 \\
\hline
\end{tabular}


Table 1 continued

\begin{tabular}{|c|c|c|c|c|c|c|}
\hline $\begin{array}{l}\text { Spot } \\
\text { number }\end{array}$ & $\begin{array}{l}\text { Gene index } \\
\text { number }\end{array}$ & $\begin{array}{l}\text { AGI gene } \\
\text { locus }\end{array}$ & Protein identity & $\mathrm{kDa} / \mathrm{pI}^{\mathrm{b}}$ & $\begin{array}{l}\text { Sequence } \\
\text { coverage }(\%)\end{array}$ & $\begin{array}{l}\text { Mascot } \\
\text { score }\end{array}$ \\
\hline 130 & 21593328 & At3g53750 & Actin $11^{\mathrm{d}}$ & $42.0 / 5.3$ & 10 & 127 \\
\hline 131 & 21593191 & At1g47980 & Dessication-related protein, putative & $34.4 / 8.7$ & 12 & 163 \\
\hline 132 & 21553354 & At2g21660 & Glycine-rich RNA binding protein 7 & $16.9 / 5.9$ & 78 & 310 \\
\hline $133^{*}$ & 21618254 & None & Unknown protein & $11.8 / 5.4$ & 73 & 74 \\
\hline 136 & 16225 & At2g27030 & Calmodulin & $16.9 / 4.2$ & 30 & 148 \\
\hline 137 & 12323093 & At3g62730 & Dessication-related protein $70055-71849$ & $33.0 / 8.3$ & 12 & 153 \\
\hline 138 & 9802567 & At1g08840 & F2203.32 (superoxide dismutase) & $14.8 / 5.4$ & 19 & 120 \\
\hline 139 & 5103841 & At1g15415 & F9L1.37 (similar to LEA) ${ }^{\mathrm{d}}$ & $10.4 / 5.9$ & 44 & 74 \\
\hline 140 & 16225 & At2g27030 & Calmodulin & $16.9 / 4.2$ & 30 & 196 \\
\hline 141 & 16223 & At1g66400 & Calmodulin & $16.9 / 4.2$ & 30 & 188 \\
\hline 142 & 21592567 & At1g80230 & Cytochrome $\mathrm{C}$ oxidase subunit & $18.9 / 5.4$ & 32 & 193 \\
\hline 143 & 21536544 & At2g19770 & Profilin 4 & $14.1 / 5.0$ & 17 & 153 \\
\hline 144 & 21553773 & At2g19760 & Profilin 3/profilin 4 & $14.6 / 5.0$ & 57 & 258 \\
\hline 145 & 28827234 & At4g13560 & LEA-domain containing protein ${ }^{\mathrm{d}}$ & $11.6 / 7.7$ & 87 & 150 \\
\hline 146 & 21537389 & At4g29340 & Profilin $3^{\mathrm{c}, \mathrm{d}}$ & $14.6 / 5.0$ & 46 & 191 \\
\hline 147 & 4115366 & At1g46696 & Unknown protein (DUF 601 conserved domain) & $47.1 / 5.9$ & 36 & 265 \\
\hline 148 & 28827234 & At4g13560 & Unknown protein (containing LEA domain) ${ }^{\mathrm{d}}$ & $11.6 / 7.7$ & 45 & 149 \\
\hline $149 \mathrm{a}$ & 55978673 & At1g04670 & Hypothetical protein ${ }^{\mathrm{d}}$ & $13.5 / 9.3$ & 26 & 89 \\
\hline $149 \mathrm{~b}$ & 71633 & At3g53750 & Actin fragment $\mathrm{d}^{\mathrm{d}}$ & $42.0 / 5.3$ & 13 & 73 \\
\hline
\end{tabular}

${ }^{a}$ Protein spot numbers with asterisk (*) were identified by MALDI-TOF MS and the rest by LC-MS/MS analysis. The annotation of spot number with ' $a$ ' and ' $b$ ' indicates that two different proteins were identified within that spot. No significant matches were found for the spot numbers not listed in the table

b Molecular mass and $\mathrm{pI}$ of identified proteins

c Proteins reported by Holmes-Davis et al. (2005)

${ }^{d}$ Proteins reported by Noir et al. (2005)

expressed in mature Arabidopsis pollen are involved in energy and general metabolism, and in defense against biotic or abiotic stresses. Studies of the Columbia ecotype revealed three spots as $\mathrm{Ca}^{2+}$. binding proteins and four spots as LEA proteins (Noir et al. 2005), and none in data provided by Holmes-Davis et al. (2005). Our study, however, revealed 11 spots representing $\mathrm{Ca}^{2+}$-binding proteins

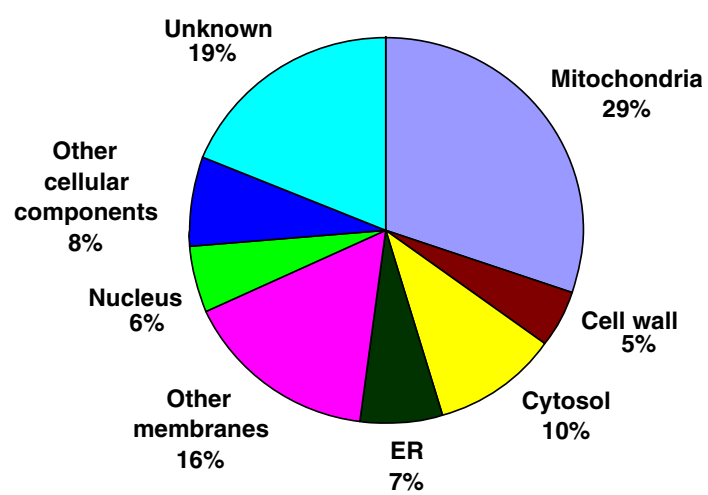

Fig. 2 The cellular component classification of identified Arabidopsis pollen proteins using gene ontology database at TAIR (http://www.arabidopsis.org/). The percentage values indicate the proportion of total number of proteins within that category and six spots as LEA proteins. Interestingly, other studies on the pollen proteome of Arabidopsis (Holmes-Davis et al. 2005; Noir et al. 2005) or rice (Dai et al. 2006) did not detect the presence of glycine-rich proteins (GRPs), whereas we identified a number of GRPs (see below). GRPs were also reported in a study on Arabidopsis pollen coat proteome (Mayfield et al. 2001), although they were

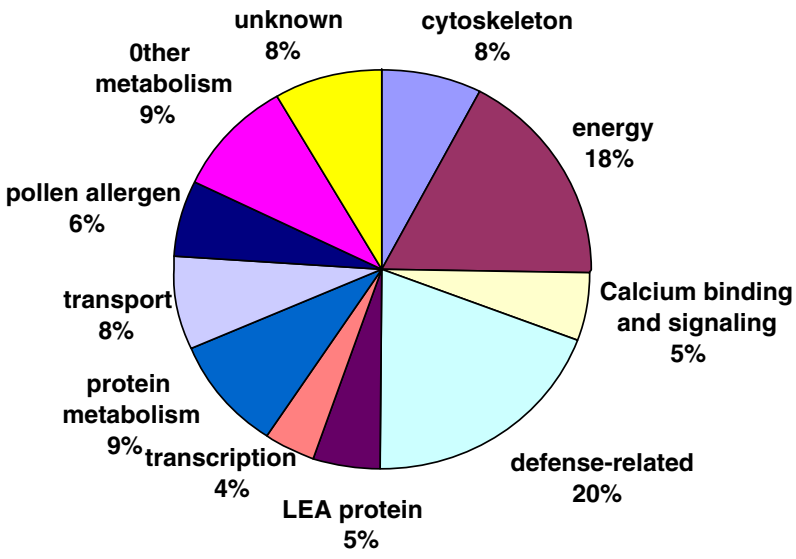

Fig. 3 Functional classification of identified proteins from mature Arabidopsis pollen. The percentage values indicate the relative proportion of total number of proteins within each group 
different from those reported here. Further, about $27 \%$ of the polypeptides reported by Noir et al. (2005) have unknown function, whereas the corresponding figure is $8 \%$ for our study, as in HolmesDavis et al. (2005). These discrepancies could be due to a number of factors including different methods of protein extraction and separation, spot selection for analysis, and different ecotypes used. That $\mathrm{Ca}^{2+}$ binding proteins were not found by Holmes-Davis et al. (2005) could be attributed to the low pI of these proteins (4-4.5), which were likely excluded from their gels. In any event, $15 \%$ of the proteins involved in general metabolism were common in all three studies.

\section{Cytoskeletal proteins}

A number of proteins were identified as actin and profilins (spots 42b, 51, 130, 143, 144, 146, and 149b), which have a role in cytoskeleton and cell organization. A functional actin cytoskeleton is a prerequisite for successful pollen germination and tube growth (Drøbak et al. 2004; Wang et al. 2004). The actin cytoskeleton coordinates the transport of vesicles containing new cell wall materials and plasma membrane to the tip of the growing pollen tube, acts as a track system for cytoplasmic streaming, and appears to be a target for calcium-mediated pollen tube growth (Drøbak et al. 2004; Wang et al. 2004; Cardenas et al. 2005). Profilin is a key actin-binding protein involved in pollen tube growth (Hepler et al. 2001) and its presence has also been reported in mature Arabidopsis (Columbia ecotype) (Holmes-Davis et al. 2005; Noir et al. 2005) and rice pollen (Dai et al. 2006). Parallel increases in actin and profilin were reported in developing anthers of rice (Kerim et al. 2003).

\section{Calcium-binding proteins}

Several proteins were identified as $\mathrm{Ca}^{2+}$-binding proteins, including calmodulin, calreticulin (CRT), and polcalcin (spots 22, 23, 37, 38, 99, 110, 112, 118, 127, 136, 140, and 141; Fig. 1, Table 1). Calcium plays an important role in pollen germination and tube growth (Malhó et al. 2000). There are complex $\mathrm{Ca}^{2+}$ homeostatic mechanisms, including pumps, ion channels, and $\mathrm{Ca}^{2+}$ buffers, involved in controlling $\mathrm{Ca}^{2+}$ transport (Iwano et al. 2004). Spots 136, 140, and 141 were identified as calmodulin, which appears to work with cyclic nucleotides to regulate the opening of ion channels, and is involved in $\mathrm{Ca}^{2+}$ signaling (Bouche et al. 2005) and pollen germination and tube growth (Golovkin and Reddy 2003). Spots 22, 23, 37, and 38 were identified as the high capacity, low affinity $\mathrm{Ca}^{2+}$ binding protein CRT. This is an ER localized protein that is important for ion buffering, and appears to coordinate $\mathrm{Ca}^{2+}$ mobilization. CRT was also reported in rice (Dai et al. 2006) and Arabidopsis pollen (Noir et al. 2005), and in Petunia pollen tubes and styles (Lenartowska, et al. 2002). The presence of CRT in Arabidopsis flowers has been demonstrated, with a proposed role in anther maturation and dehiscence (Nelson et al. 1997), in addition to its role in $\mathrm{Ca}^{2+}$ binding, and as a molecular chaperone. A putative $\mathrm{Ca}^{2+}$-binding protein (spot 99) and a calmodulinbinding protein (spot 20) have also been identified, and these may have unspecified roles in $\mathrm{Ca}^{2+}$ signal transduction in the pollen during germination.

Synaptosomal-associated protein (SNAP-25) was identified (spots 49 and 50) as a plasma membrane associated protein involved in $\mathrm{Ca}^{2+}$ transport and the activation and maintenance of store-mediated $\mathrm{Ca}^{2+}$ entry (Chieregatti et al. 2004). This protein has a role in vesicle-mediated transport and protein-mediated disease resistance (Collins et al. 2003). The presence of a large number of $\mathrm{Ca}^{2+}$-binding proteins in the mature pollen is in support of their requirement for germination and tube growth.

\section{Defense-related proteins}

Defense-related proteins include resistance proteins, defense-regulated proteins, proteins involved in apoptosis, cell rescue, stress response, and detoxification. Many of the plant responses to external factors, e.g., pathogen attack, are related to the production of reactive oxygen species (ROS). Recent evidence suggests that ROS function as cellular second messengers that modulate many different proteins leading to a variety of responses (Foyer and Noctor 2005). However, the excess production of ROS under biotic and abiotic stresses causes oxidative damage to cellular compartments (Apel and Hirt 2004). Plants combat oxidative stress by inducing various protective enzymes and anti-oxidants. In Arabidopsis pollen, a number of protein spots involved in detoxification of ROS were identified: i.e., superoxide dismutase (spots 80 and $138)$, ascorbate peroxidase $(68,70,87$, and 101), dehydro-ascorbate reductase ( 82 and 91 ), glutathione transferase (74 and 113), phospholipid hydroperoxide glutathione peroxidase (106 and 117), lipoxygenase (126), and alkyl hydroperoxide reductase (95 and 96a) (Table 1). All these enzymes are known to play a crucial role in oxidative stress and the ascorbateglutathione cycle in plants (Chew et al. 2003), and their presence in the pollen may provide protection against 
oxidative stress. Most of these enzymes were also reported in Arabidopsis (Columbia ecotype) (HolmesDavis et al. 2005; Noir et al. 2005) and rice pollen (Dai et al. 2006).

Several luminal binding proteins (BiP, spots $8,31,56$, 57, 71, 72, and 107), and protein disulfide isomerases (PDI, spots 13, 21, 46, 53, 58, 59, and 60) belonging to the thioredoxin family, were also identified. These proteins are localized in ER and are known as molecular chaperones involved in proper protein folding, a necessary process for protein transport. Under stress, the folding or assembly of proteins is disordered and this is associated with enhanced expression of genes such as $B i P$ and PDI (Koizumi et al. 2001). PDI has been implicated in fungal pathogen resistance in wheat (Ray et al. 2003). The presence of PDI and BiP in Arabidopsis and rice pollen (Dai et al. 2006) may reflect their role in stress tolerance during pollen maturation.

Protein phosphatase $2 \mathrm{C}$, an enzyme that belongs to a class of ubiquitous and evolutionarily conserved serine/threonine protein phosphatases, was identified (spot 109). This enzyme acts as a negative regulator of stress/abscisic acid (ABA) signaling (Zhang et al. 2004). Since ABA is known to play a role in seed and bud dormancy (Zeevaart and Creelman 1988), protein phosphatase 2C may be important for breaking pollen dormancy at the time of germination. Protein spots 131 and 137 were identified as desiccation-related proteins and spot 10 as heat shock protein. Since the pollen grain is an extremely desiccated structure at maturity, these proteins may serve to protect the cytoplasmic contents in the pollen. Desiccation-related proteins were also reported in the Arabidopsis Columbia ecotype pollen (Noir et al. 2005).

A number of LEA-like proteins or proteins containing the LEA domain were identified in Arabidopsis pollen (spots 39, 52, 75a, 83, 114, 139, and 148). LEA proteins have been shown to accumulate during seed maturation, and their expression has been linked to the acquisition of desiccation tolerance. Many LEA proteins are induced by cold or osmotic stress, or by exogenous ABA, or are expressed constitutively (Wang et al. 2003). Though the precise function of LEA is not known, recent transgenic studies suggest their roles include stress tolerance (Park et al. 2005) and protection of enzyme activity and protein aggregation under water stress (Goyal et al. 2005). LEA-like proteins in Arabidopsis pollen may thus provide protection against desiccation during pollen maturation in addition to other unknown functions. LEA-like proteins were also reported in one other study on Arabidopsis pollen (Noir et al. 2005) and in lily pollen (Miki-Hirosige et al. 2004), but not in rice pollen (Dai et al. 2006).
Another group of proteins identified in Arabidopsis pollen were GRPs (spots 24, 86, 108, 123, 124, and 132; Table 1). Three of these were identified as the RNAbinding proteins AtGRP8 and AtGRP7, and a fourth as the cell wall protein GRP2. GRPs have been implicated in post-transcriptional regulation of gene expression, including RNA processing, which is involved in developmental regulation in plants (SachettoMartins et al. 2000). The expression levels of many GRPs have been shown to be up-regulated by various stress conditions, particularly cold and salinity, and they play an important role in defense (Kim et al. 2005). The presence of GRPs in Arabidopsis pollen at maturity may, therefore, provide another shield against environmental stresses. A group of lipid-binding GRPs known as oleosins were reported in Arabidopsis pollen coat (Mayfield et al. 2001).

Pollen allergens

Pollen grains are known to have a number of proteins which act as allergens (Mohapatra and Knox 1996). Spots 110, 112, and 118 were identified as polcalcins, which are known to act as allergens in a number of species in addition to their role in $\mathrm{Ca}^{2+}$-binding. Other proteins identified in Arabidopsis pollen, e.g., profilins, $\mathrm{BiP}$, and calcium binding proteins, also act as allergens (Radauer and Breiteneder 2006).

\section{Energy-related proteins}

A number of proteins identified were those involved in energy conversion reactions. These proteins are mainly associated with electron transport in addition to glycolysis, pentose-phosphate-pathway, and TCA-cycle. The pollen grain, like the seed, requires energy during germination and tube growth, and it appears that the mature pollen has this energy machinery in place at the time of maturity. Many studies have shown the presence of enzymes in pollen that participate in energy conversion, general cell maintenance, and metabolism (HolmesDavis et al. 2005; Noir et al. 2005; Dai et al. 2006). The Arabidopsis pollen transcriptome studies have also shown a number of transcripts related to the enzymes involved in energy metabolism (Honys and Twell 2003).

\section{Other proteins}

It is known that pollen germination is largely dependent on translation of pre-synthesized mRNAs (Mascarenhas 1990). Therefore, translational control and signal transduction play an important role in pollen germination and tube growth (Honys and Twell 2003). 
Spot 125 was identified as translational initiation factor 5A-2 (eIF-5A 2), and spot 129 as 60S ribosomal protein involved in translational elongation. These proteins could, therefore, potentially be important for translation of stored mRNA during pollen germination. The translation initiation factor $5 \mathrm{~A}$ was identified in rice pollen (Dai et al. 2006), and a putative translation initiation factor transcript is known to be selectively expressed in Arabidopsis pollen (Honys and Twell 2003). A number of proteins involved in protein transport within the cell were also identified.

Spot $103 \mathrm{~b}$ was identified as a proteosome multicatalytic endopeptidase known to eliminate damaged or unneeded proteins and to participate in regulation by targeting and degrading short-lived regulatory proteins (Coux et al. 1996). Spots 111 and 116 were pectin methylesterase (PME) inhibitors, which play an important role in post-translational modification of PME, and were also reported in other studies on Arabidopsis and rice pollen (Holmes-Davis et al. 2005; Noir et al. 2005; Dai et al. 2006). PME also has roles in fruit ripening, microsporogenesis, pollen tube growth, seed germination, and hypocotyl elongation (Pilling et al. 2004).

\section{Unknown proteins}

Spots 5, 6, 25, 30, 47, 133, 147, and 149a, were identified as hypothetical/unknown or expressed proteins from Arabidopsis database, with no well-defined function. Spot 47 contains a clathrin light chain domain, which is a major vesicle coat protein. It is possible that this protein might participate in vesicle formation during pollen tube growth. The identified protein in spot 133 has no corresponding transcript in the database. A large number of transcripts (Honys and Twell 2003) and proteins reported in Arabidopsis and rice pollen (Holmes-Davis et al. 2005; Noir et al. 2005; Dai et al. 2006) also belong to the group with unknown function.

Many Arabidopsis pollen proteins are similar to pollen transcripts

Arabidopsis pollen transcriptome studies reported that approximately $10 \%$ of transcripts are pollen-specific (Honys and Twell 2003). Our proteome analysis has shown that $12 \%$ of the identified proteins were pollenspecific, i.e., actin, profilins, calmodulin, initiation factor $5 \mathrm{~A}$, and a hypothetical protein, based upon their corresponding pollen specific transcripts. Thus, in addition to the presence of transcripts, a number of proteins needed for germination and tube growth are in place at the time when pollen is released from the anther. Many of the proteins involved in energy and defense-related mechanisms, including stress related proteins, were also similar to the reported transcripts (Honys and Twell 2003; Pina et al. 2005). However, the frequency of occurrence of abundant proteins has an inverse relationship with the transcript level (HolmesDavis et al. 2005).

Arabidopsis pollen has a number of proteins similar to those in seeds

The pollen grain at maturity, like the seed, is a dormant, desiccated structure and both are dispersal agents in higher plants. Although the pollen grain is a haploid gametophytic tissue and the seed a diploid sporophytic tissue, it is interesting to note that the pollen has a number of proteins similar to those in seed. A comparison of the pollen proteome with that of seed proteome of Arabidopsis (Gallardo et al. 2001), wheat (Vensel et al. 2005), and tomato (Sheoran et al. 2005) indicates that many proteins, e.g., LEA, actin, profilins, dismutase, dihydroascorbate reductase, enolase, PDI, BiP, and ATP synthase, are common to both these structures. LEA proteins likely play a role in stress tolerance during desiccation of pollen and the seed, and actin and profilins would contribute to the formation of the cytoskeleton during germination and growth of both these structures. Other similar proteins and enzymes in the pollen and seed would be required in metabolic processes. One of the striking differences between the two structures was the lack of storage proteins identified in the pollen, e.g., legumins, crucifirin, and vicilins, which are abundant in the seeds. The transcriptome analysis of Arabidopsis pollen also revealed the absence of storage protein-related transcripts (Becker et al. 2003; Honys and Twell 2003; Pina et al. 2005). This may be due to the fact that the seed, upon germination, requires stored proteins for extensive meristematic activity to form a young seedling with a root and shoot system. In contrast, pollen germination and tube growth mainly involve extension of the vegetative cell and, therefore, the requirement of stored proteins may be limited.

\section{Conclusions}

This study on the proteome analysis of Arabidopsis pollen reports on a number of proteins not reported earlier, i.e., calcium binding proteins, GRPs, LEA-like proteins, various isoforms of $\mathrm{MDH}$, transcription factor, initiation factor, protein phosphatase $\mathrm{C}$, and profilin 4 . However, many proteins identified were similar to two 
other studies on Arabidopsis pollen. Our study was on the Landsberg erecta ecotype in contrast to other studies on the Columbia ecotype and this, along with differences in the methods of protein extraction and spot selection, would explain the differences in the pollen proteins between these studies. Nevertheless, together, these three studies on the proteome of two different ecotypes of Arabidopsis advance our knowledge on the nature and function of proteins present in the pollen grain at maturity. The proteome of Arabidopsis pollen is, however, not yet fully analyzed and further work is needed to identify additional proteins, especially the low abundance proteins, and their roles in pollen.

Acknowledgment This research was supported by a Discovery grant from NSERC of Canada to V. K. S.

\section{References}

Apel K, Hirt H (2004) Reactive oxygen species: metabolism, oxidative stress, and signal transduction. Annu Rev Plant Biol 55:373-399

Becker JD, Boavida LC, Carneiro J, Haury M, Feijó JA (2003) Transcriptional profiling of Arabidopsis tissues reveals the unique characteristics of the pollen transcriptome. Plant Physiol 133:713-725

Bedinger P (1992) The remarkable biology of pollen. Plant Cell 4:879-887

Berardini TZ, Mundodi S, Reiser R, Huala E, Garcia-Hernandez M, Zhang P, Mueller LM, Yoon J, Doyle A, Lander G, Moseyko N, Yoo D, Xu I, Zoeckler B, Montoya M, Miller N, Weems D, Rhee SY (2004) Functional annotation of the Arabidopsis genome using controlled vocabularies. Plant Physiol 135:745-755

Bouche N, Yellin A, Snedden WA, Fromm H (2005) Plantspecific calmodulin-binding proteins. Annu Rev Plant Biol 56:435-466

Candiano G, Bruschi M, Musante L, Santucci L, Ghiggeri GM, Carnemolla B, Orecchia P, Zardi L, Righetti PG (2004) Blue silver: a very sensitive colloidal Coomassie G-250 staining for proteome analysis. Electrophoresis 25:13271333

Cardenas L, Lovy-Wheeler A, Wilsen KL, Hepler PK (2005) Actin polymerization promotes the reversal of streaming in the apex of pollen tubes. Cell Motil Cytoskeleton 61:112127

Chew O, Whelan J, Millar AH (2003) Molecular definition of the ascorbate-glutathione cycle in Arabidopsis mitochondria reveals dual targeting of antioxidant defenses in plants. J Biol Chem 278:46869-46877

Chieregatti E, Chicka MC, Chapman ER, Baldini G (2004) SNAP-23 functions in docking/fusion of granules at low Ca2+. Mol Biol Cell 15:1918-1930

Collins NC, Thordal-Christensen H, Lipka V, Bau S, Kombrink E, Qiu JL, Huckelhoven R, Stein M, Freialdenhoven A, Somerville SC, Schulze-Lefert P (2003) SNARE-proteinmediated disease resistance at the plant cell wall. Nature 425:973-977

Coux O, Tanaka K, Goldberg A (1996) Structure and functions of the $20 \mathrm{~S}$ and $26 \mathrm{~S}$ proteasomes. Annu Rev Biochem 65:801-847
Dai S, Li L, Chen T, Chong K, Xue Y, Wang T (2006) Proteomic analysis of Oryza sativa mature pollen reveal novel proteins associated with pollen germination and tube growth. Proteomics 6:2504-2529

Drøbak BK, Franklin-Tong VE, Staiger CJ (2004) The role of the actin cytoskeleton in plant cell signaling. New Phytol 163:13-30

Engel ML, Chaboud A, Dumas C, McCormick S (2003) Sperm cells of Zea mays have a complex complement of mRNAs. Plant J 34:697-707

Fei H, Zhang R, Pharis RP, Sawhney VK (2004) Pleiotropic effects of the male sterile33 (ms33) mutation in Arabidopsis are associated with modification in endogenous gibberellins indole-3-acetic acid and abscisic acid. Planta 219:649-660

Foyer CH, Noctor G (2005) Oxidant and antioxidant signaling in plants: a re-evaluation of the concept of oxidative stress in a physiological context. Plant Cell Environ 28:1056-1071

Gallardo K, Job C, Groot SPC, Puype M, Demol H, Vandekerckhove J, Job D (2001) Proteomic analysis of Arabidopsis seed germination and priming. Plant Physiol 126:835-848

Golovkin M, Reddy ASN (2003) Calmodulin-binding protein from Arabidopsis has an essential role in pollen germination. Proc Natl Acd Sci USA 100:10558-10563

Goyal K, Walton LJ, Tunnacliffe A (2005) LEA proteins prevent protein aggregation due to water stress. Biochem J 388:151157 (part 1)

Hamilton DA, Mascarenhas JP (1997) Gene expression during pollen development. In: Shivanna KR, Sawhney VK (eds) Pollen biotechnology for crop production and improvement. Cambridge University press, UK, pp 40-58

Hanson MR, Betolila S (2004) Interactions of mitochondrial and nuclear genes that affect male gametophyte development. Plant Cell 16:S154-S169

Hepler PK, Vidali L, Cheung AY (2001) Polarized cell growth in higher plants. Annu Rev Cell Dev Biol 17:159-187. DOI 10.1146/annurev.cellbio.17.1.159

Holmes-Davis R, Tanaka CK, Vensel WH, Hurkman WJ, McCormick S (2005) Proteome mapping of mature pollen of Arabidopsis thaliana. Proteomics 5:4864-4884

Honys D, Twell D (2003) Comparative analysis of the Arabidopsis pollen transcriptome. Plant Physiol 132:640-652

Iwano M, Shiba H, Miwa T, Che FS, Takayama S, Nagai T, Miyawaki A, Isogai A (2004) $\mathrm{Ca}^{2+}$ dynamics in a pollen grain and papilla cell during pollination of Arabidopsis. Plant Physiol 136:3562-3571

Kerim T, Imin N, Weinman JJ, Rolfe BG (2003) Proteome analysis of male gametophyte development in rice anthers. Proteomics 3:738-751

Kim YO, Kim JS, Kang H (2005) Cold-inducible zinc fingercontaining glycine-rich RNA-binding proteins contributes to the enhancement of freezing tolerance in Arabidopsis thaliana. Plant J 42:890-900

Koizumi N, Martinez IM, Kimata Y, Kohno K, Sano H, Chrispeels MJ (2001) Molecular characterization of two Arabidopsis Ire 1 homologs, endopasmic reticulum-located transmembrane protein kinases. Plant Physiol 127:949-962

Lenartowska M, Karas K, Marshall J, Napier R, Bednarska E (2002) Immunocytochemical evidence of Calreticulin-like protein in pollen tubes and styles of Petunia hybrida Hort. Protoplasma 219:23-30

Malho R, Camacho L, Moutinho A (2000) Signaling pathways in pollen tube growth and reorientation. Ann Bot 85:59-68

Mascarenhas JP (1990) Gene activity during pollen development. Annu Rev Plant Physiol Plant Mol Biol 41:317-338 
Mayfield JA, Fiebig A, Johnstone SE, Preuss D (2001) Gene families from the Arabidopsis thaliana pollen coat proteome. Science 292:2482-2485

McCormick S (2004) Control of male gametophyte development. Plant Cell 16:S142-S153

Miki-Hirosige H, Yamanaka Y, Nakamura S, Kurata S, Hirano $\mathrm{H}$ (2004) Changes of protein profiles during pollen development in Lilium longiflorum. Sex Plant Reprod 16:209-214

Mohapatra SS, Knox RB (1996) Pollen biotechnology: gene expression and allergen characterization. Chapman and Hall, New York

Nelson DE, Glaunsinger B, Bohnert HJ (1997) Abundant accumulation of the calcium-binding molecular chaperone calreticulin in specific floral tissues of Arabidopsis thaliana. Plant Physiol 114:29-37

Noir S, Brautigam A, Colby T, Schmidt J, Panstruga R (2005) A reference map of the Arabidopsis thaliana mature pollen proteome. Biochem Biophys Res Commun 337:1257-1266

Palmer RG, Albertsen MC, Horner HT, Skorupska H (1992) Male sterility in soybean and maize: developmental comparisons. Nucleus (Calcutta) 35:1-18

Park BJ, Liu ZC, Kanno A, Kameya T (2005) Increased tolerance to salt- and water-deficit stress in transgenic lettuce (Lactuca sativa L.) by constitutive expression of LEA. Plant Growth Regul 45:165-171

Pilling J, Willmitzer L, Bucking H, Fisahn J (2004) Inhibition of a ubiquitously expressed pectin methyl esterase in Solanum tuberosum L. affects plant growth, leaf growth polarity, and ion portioning. Planta 219:32-40

Pina C, Pinto F, Feijo JA, Becker JD (2005) Gene family analysis of the Arabidopsis pollen transcriptome reveals biological implications for cell growth, division control, and gene expression regulation. Plant Physiol 138:744-756

Radauer C, Breiteneder H (2006) Pollen allergens are restricted to few protein families and show distinct patterns of species distribution. J Allergy Clin Immunol 117:141-147

Ray S, Anderson JM, Urmeev FI, Goodwin SB (2003) Rapid induction of a protein disulfide isomerase and defenserelated genes in wheat in response to the hemibiotrophic fungal pathogen Mycosphaerella graminicola. Plant Mol Biol 53:741-754

Roulston TH, Cane JH, Buchmann SL (2000) What governs protein content of pollen: pollinator preferences, pollenpistil interactions, or phylogeny?. Ecol Monogr 70:617-643
Russell SD, Strout GW (2005) Microgametogenesis in Plumbago Zeylanica (Plumbaginaceae). 2. Quantitative cell and organelle dynamics of the male reproductive cell lineage. Sex Plant Reprod 18:113-130

Sachetto-Martins G, Franco LO, de Oliveira DE (2000) Plant glycine-rich proteins: a family or just proteins with a common motif?. Biochim Biophys Acta 1492:1-14

Sanders PM, Bui AQ, Weterings K, McIntire KN, Hsu Y-C, Lee PY, Truong MT, Beals TP, Goldberg RB (1999) Anther developmental defects in Arabidopsis thaliana male-sterile mutants. Sex Plant Reprod 11:297-322

Scott RJ, Spielman M, Dickinson HG (2004) Stamen structure and function. Plant Cell 16:S46-S60

Sheoran IS, Olson DJH, Ross ARS, Sawhney VK (2005) Proteome analysis of embryo and endosperm of germinating tomato seeds. Proteomics 5:3752-3764

Shivanna KR, Sawhney VK (1995) Polyethylene glycol improves the in vitro growth of Brassica pollen tubes without loss of germination. J Exp Bot 46:1771-1774

Vensel WH, Tanaka CK, Cai N, Wong JH, Buchanan BB, Hurkman WJ (2005) Developmental changes in the metabolic protein profiles of wheat endosperm. Proteomics 5:1594-1611

Wang WX, Vinocur B, Altman A (2003) Plant responses to drought, salinity and extreme temperatures: towards genetic engineering for stress tolerance. Planta 218:1-14

Wang YF, Fan LM, Zhang WZ, Zhang W, Wu WH (2004) Ca2+ permeable channels in the plasma membrane of Arabidopsis pollen are regulated by actin microfilaments. Plant Physiol 136:3892-3904

Xu HL, Swoboda I, Bhalla PL, Singh MB (1999) Male gametic cell-specific gene expression in flowering plants. Proc Natl Acad Sci USA 96:2554-2558

Zeevaart JAD, Creelman RA (1988) Metabolism and physiology of abscisic-acid. Annu Rev Plant Physiol Plant Mol Biol 39:439-473

Zhang WH, Qin CB, Zhao J, Wang XM (2004) Phospholipase D alpha-1 derived phosphatic acid interacts with ABI 1 phosphatase $2 \mathrm{C}$ and regulates abscisic acid signaling. Proc Natl Acad Sci USA 101:9508-9513 